\title{
X. On aerial waves generated by impact. Part II
}

\section{Sudhansukumar Banerji M.Sc.}

To cite this article: Sudhansukumar Banerji M.Sc. (1918) X. On aerial waves generated by impact. Part II, Philosophical Magazine Series 6, 35:205, 97-111, DOI: 10.1080/14786440108635739

To link to this article: http://dx.doi.org/10.1080/14786440108635739

$$
\text { 曲 Published online: } 08 \text { Apr } 2009 .
$$

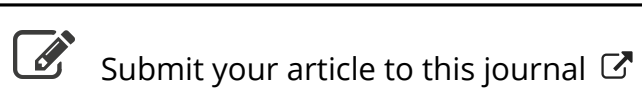

\section{Џll Article views: 4}

Q View related articles $\asymp$

4 Citing articles: 1 View citing articles 준 
they may be entirely separated. When this is the case, the fringes of the ordinary Fresnel type due to the edge of the cylinder may be observed, and inside the shadow we have also an entirely separate system of fringes due to the reflected rays, the first and principal maximum of which lies alongside the virtual caustic formed by oblique reflexion; the distribution of intensity in this system can be found from the well-known integral due to Airy; (d) but when the focal plane is only a little in advance of the edge, the caustic and the reflecting surface are nearly in contact, and Airy's investigation of the intensity in the neighbourhood of a caustic requires modification. It is then found that only a finite number of bands (one, two, three, or more according to the position of tho plane of observation) is formed within the limits of the shadow, and not an indefnitely large number as contemplated by Airy's theory. The rest of the fringes seen in the field are due to the interference of the direct and reflected rays, but modified by diffraction at the edge of the cylinder.

The Indian Association

for the Cultivation of Science,

Calcutta, 8th May, 1917.

X. On Aerial Waves generated by Impact. Part II. By Sudhansurgmar Banerui, M.Sc., Assistant Professor of Applied Mathematics, Uriversity of Calcutta* .

[Plate IV.]

\section{Introduction.}

THE origin and characteristics of the sound produced by the collision of two solid spheres were discussed by me at some length in the first paper under the same title that was published in the Philosophical Magazine for July, 1916. It was shown in that paper that the sound is not due to the vibrations set up in the spheres, which in any ordinary material are both too high in pitch to be audible and too faint in intensity, but to aerial waves set up by the reversal of the motion of the spheres as a whole. The intensity of the sound in different directions for the case in which the two spheres were of the same material and diameter, was investigated by the aid of a new instrument which will be referred to as

* Communicated by Prof. C. V. Raman.

Phil. Mag. S. 6. Vol. 35. No. 205. Jan. 1918. 
"the ballistic phonometer *." The intensity was found to be a maximum along the line of collision, falling off gradually in other directions to a value which is practically zero on the surface of a cone of semi-vertical angle $67^{\circ}$, and rising again to a second but feebler maximum in a plane at right angles to the line of collision.

In viow of the interesting results obtained for the case of two equal spheres, it was arranged to continue the investigation and to measure the distribution of intensity when the colliding spheres were not both of the same radius or material. A mathematical investigation of the nature of the results to be expected in these cases was also undertaken. In order to exhibit the results of the measurements and of the theoretical calculation, a plan has now been adopted which is much more suitable than the one used in the first paper. This will be best understood by reference to fig. 1 (Pl. IV.), which refers to the case of two spheres of the same material and diameter. The figure has been drawn by taking the point at which the spheres impinge as origin, and the line of collision as the axis of $x$, and setting off the indications of the ballistic phonometer as radii vectores at the respective angles which the directions in which the sound is measured make with the line of collision. The curve thus represents the distribution of intensity round the colliding spheres in polar coordinates, the points at which the intensity of the sound is measured being assumed to be all at the same distance from the spheres. The results are brought much more vividly before the eye by a diagram of this kind than by plotting the results on squared paper.

\section{Case of two spheres of the same material but of different diameters.}

Fig. 2, which shows the observed distribution of intensity when two spheres of wood of diameters 3 inches and $2 \frac{1}{4}$ inches collide with each other, is typical of the results obtained when the impinging spheres are nearly of the same density and are of different diameters. There is a distinct asymmetry about a plane perpendicular to the line of impact. In addition to the maxima of intensity in the two directions of the line of collision, we have the maxima in lateral directions, which are not at right angles to this line. The

* This name was suggested by Prof. E. H. Barton, D.Sc., F.R.S., writing in the 'Science Abstracts,' p. 399, Sept. 1916. 
directions in which the intensity is a minimum are also asymmetrically situated.

For the explanation of these and other results, we have naturally to turn to the mathematical theory which rests upon the fact that the sound is due to the wave-motion set up in the fluid by the sudden reversal of the motion of the spheres. Let $a$ and $b$ be the radii of the two spheres and $\rho_{a}$ and $\rho_{b}$ be their densities. Then the masses of the spheres are $\frac{4}{3} \pi \rho_{a} a^{3}$ and $\frac{4}{3} \pi \rho_{b} b^{3}$ respectively. Denoting the changes in velocity which the balls undergo as a result of the impact by $\mathrm{U}_{a}$ and $\mathrm{U}_{b}$ respectively, by the principle of constant momentum we have $\mathrm{U}_{a} / \mathrm{U}_{b}=\rho_{b} b^{3} / \rho_{a} a^{3}$. The ratio $\frac{\mathrm{U}_{a}}{\mathrm{U}_{l}}$ thus depends only on the diameters and the densities of the spheres, while, of course, the actual values of $\mathrm{U}_{a}$ and $\mathrm{U}_{b}$ would depend on the relative velocity before impact and the coefficient of restitution. It is obvious that if we leave out of account the duration of impact, that is, regard the changes in velocity of the spheres as taking place practically instantaneously, the character and the ratio of the intensities of the sound produced in different directions would be completely determined by the sizes of the spheres and the ratio of their changes of velocity, that is, by their diameters and their masses; when the spheres are of the same material, the nature of the motion in the fluid set up by the impact would depend only on the radii of the spheres.

The complete mathematical problem of finding the nature of the fluid motion set up by the reversal of the motion of the spheres, taking the finite duration of impact into account, would appear to be of great difficulty. In my first paper, I have shown that when a single sphere of radius $a$ undergoes an instantaneous change of velocity $U$, the wave-motion produced is given by the expression

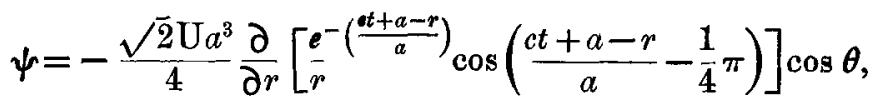

which indicates that it is of the damped harmonic type, confined to a small region near the front of the advancing wave. The wave-motion set up in the case of two spheres in contact assumed to undergo instantaneous changes of velocity would be of a more complicated type. In order to obtain a general idea of the results to be expected, particularly as to the intensity and character of the sound in 
different directions, we may consider the analogous acoustical problem of two rigid spheres nearly in contact, which execute small oscillations to and fro on the line of their centres. This problem may be mathematically formulated and approximately solved in the following mannor :-

Given prescribed vibrations

$$
\mathrm{U}_{a} \cos \theta_{1} \cdot e^{i k c t} \text { and } \mathrm{U}_{b} \cos \theta_{2} \cdot e^{i k c t}
$$

on the surfaces of two spheres of radii $a$ and $b$ nearly in contact, it is required to determine the velocity potential of the wave-motion started and the distribution of intensities round the spheres, where $\theta_{1}$ and $\theta_{2}$ are the angles measured at the centres $A$ and $B$ of the two spheres in opposite senses from the line joining the centres.

Supposing, now, that an imaginary sphere is constructed which is of just sufficient radius to envelop the two actual spheres (touching them externally), it is possible from a consideration of the nature of the motion that takes place in the immediate neighbourhood of the two spheres, to determine the aerial vibration on the surface of the imaginary sphere which would produce on the external atmosphere the same effect as the vibrations on the surfaces of the real spheres $A$ and $B$. When the equivalent vibration on the surface of the enveloping sphere has been obtained, we can, by the use of the well-known solution for a single sphere, at once determine the wave-motion at any external point.

The radius of the enveloping sphere is evidently $a+b$, and its centre is at a point $\mathrm{C}$, such that $\mathrm{BC}=a$ and $\mathrm{CA}=b$.

If the point $\mathrm{C}$ be taken as origin, and if the equivalent vibration on the surface of the enveloping sphere be expressed by the series

$$
\Sigma \mathrm{A}_{n} \mathrm{P}_{n}(\cos \theta) e^{i k c t}, \quad . \quad . \quad . \quad .
$$

where $A_{n}$ 's are known constants, the velocity potential of the wave-motion is given by

$$
\psi=-\frac{(a+b)^{2}}{r} e^{i k(c t-r+a+b)} \Sigma \frac{\mathrm{A}_{n} \mathrm{P}_{n}(\cos \theta)}{\mathrm{F}_{n}(i k \cdot \overline{a+b})} f_{n}(i k r), .
$$

where

$$
\begin{gathered}
f_{n}(i k r)=1+\frac{n(n+1)}{2 . i k r}+\frac{(n-1) n(n+1)(n+2)}{2.4 \cdot(i k r)^{2}}+\ldots \\
\ldots+\frac{1.2 .3 \ldots 2 n}{2.4 .6 \ldots 2 n \cdot(i k r)^{n}}, \\
\mathrm{~F}_{n}(i k r)=(1+i k r) f_{n}(i k r)-i k r f_{n}^{\prime}(i k r) .
\end{gathered}
$$


To obtain the equivalent vibrations on the surface of the imaginary enveloping sphere, we shall regard the small quantity of fluid enclosed by this sphere as practically incompressible, and use the well-known solution by the method of successive images for two spheres in an incompressible fluid.

We know that the velocity potential due to such a system of two spheres in an incompressible fluid can be expressed in the form

$$
\mathrm{U} \phi+\mathrm{U}_{b} \phi^{\prime}, \text {. • • • • • . }
$$

where $\phi$ and $\phi^{\prime}$ are to be determined by the conditions

$$
\begin{gathered}
\nabla^{2} \phi=0, \quad \nabla^{2} \phi^{\prime}=0, \\
\frac{\partial \phi}{\partial r_{1}}=-\cos \theta_{1}, \quad \text { and } \quad \frac{\partial \phi^{\prime}}{\partial r_{2}}=0, \text { when } r_{1}=a, \\
\frac{\partial \phi^{\prime}}{\partial r_{2}}=-\cos \theta_{2}, \quad \text { and } \frac{\partial \phi}{\partial r_{2}}=0, \quad \text { when } r_{2}=b,
\end{gathered}
$$

$r_{1}, r_{2}$ being radii vectores drawn from $\mathrm{A}$ and $\mathrm{B}$.

When $\phi$ and $\phi^{\prime}$ have been determined so as to satisfy these conditions, the equivalent vibrations on the surface of the imaginary sphere can be taken to be very approximately given by the expression

$$
-\left[\mathrm{U}_{a} \frac{\partial \phi}{\partial r}+\mathrm{U}_{b} \frac{\partial \phi^{\prime}}{\partial r}\right] e_{r=a+b}^{i k e t} \cdot . . . .
$$

The functions $\phi$ and $\phi^{\prime}$, as is well known, can be determined by the method of successive images, and if the expressions for the velocity potential due to these images be all transferred to the coordinates $r, \theta$ referred to the centre $\mathrm{C}$ of the enveloping sphere, we easily obtain

$$
\begin{aligned}
& 2 \phi=a^{3}\left[1-\frac{b^{3}}{(a+b)^{3}}\right.+\frac{b^{3}}{(a+2 b)^{3}}-\frac{b^{3}}{(2 a+2 b)^{3}} \\
&\left.+\frac{b^{3}}{(2 a+3 b)^{3}}-\ldots\right] \frac{\mathrm{P}_{1}(\cos \theta)}{r^{2}} \\
&+2 a^{3}\left[b-\frac{b^{3}\left(a^{2}+a b-b^{2}\right)}{(a+b)^{4}}+\frac{b^{3}\left(2 b^{3}-a^{2}\right)}{(a+2 b)^{4}}-\frac{b^{3}\left(2 a^{2}+a b-2 b^{2}\right)}{(2 a+2 b)^{4}}\right. \\
&\left.+\frac{b^{3}\left(3 b^{2}-2 a^{2}\right)}{(2 a+3 b)^{4}}-\ldots\right] \frac{\mathrm{P}_{2}(\cos \theta)}{r^{3}}
\end{aligned}
$$




$$
\begin{aligned}
&+3 a^{3}\left[b^{2}-\frac{b^{3}\left(a^{2}+a b-b^{2}\right)^{2}}{(a+b)^{5}}\right.+\frac{b^{3}\left(2 b^{2}-a^{2}\right)^{2}}{(a+2 b)^{5}}-\frac{b^{3}\left(2 a^{2}+a b-2 b^{2}\right)^{2}}{(2 a+2 b)^{5}} \\
&\left.+\frac{b^{3}\left(3 b^{2}-2 a^{2}\right)^{2}}{(2 a+3 b)^{5}}-\ldots\right] \frac{\mathrm{P}_{3}(\cos \theta)}{r^{4}} \\
&+4 a^{3}\left[b^{3}-\frac{b^{3}\left(a^{2}+a b-b^{2}\right)^{3}}{(a+b)^{6}}+\frac{b^{3}\left(2 b^{2}-a^{2}\right)^{3}}{(a+2 b)^{8}}-\frac{b^{3}\left(2 a^{2}+a b-2 b^{2}\right)^{3}}{(2 a+2 b)^{6}}\right.
\end{aligned}
$$$$
\left.+\frac{b^{3}\left(3 b^{2}-2 a^{2}\right)^{3}}{(2 a+3 b)^{6}}-\ldots\right] \frac{\mathbf{P}_{4}(\cos \theta)}{r^{5}}
$$$$
+ \text { \&c., }
$$

and

$$
\begin{aligned}
& 2 \phi^{\prime}=-b^{3}\left[1-\frac{a^{3}}{(b+a)^{3}}+\frac{a^{3}}{(b+2 a)^{3}}-\frac{a^{3}}{(2 b+2 a)^{3}}\right. \\
& \left.+\frac{a^{3}}{(2 b+3 a)^{3}}-\ldots\right] \frac{\mathrm{P}_{1}(\cos \theta)}{r^{2}} \\
& +2 b^{3}\left[a-\frac{a^{8}\left(b^{2}+a b-a^{2}\right)}{(b+a)^{4}}+\frac{a^{3}\left(2 a^{2}-b^{2}\right)}{(b+2 a)^{4}}-\frac{a^{3}\left(2 b^{2}+a b-2 a^{2}\right)}{(2 b+2 a)^{4}}\right. \\
& \left.+\frac{a^{3}\left(3 a^{2}-2 b^{2}\right)}{(2 b+3 a)^{4}}-\ldots\right] \frac{\mathrm{P}_{2}(\cos \theta)}{r^{3}} \\
& -3 b^{3}\left[a^{2}-\frac{a^{3}\left(b^{2}+a b-a^{2}\right)^{2}}{(b+a)^{5}}+\frac{a^{3}\left(2 a^{2}-b^{2}\right)^{2}}{(b+2 a)^{5}}-\frac{a^{3}\left(2 b^{2}+a b-2 a^{2}\right)^{2}}{(2 b+2 a)^{5}}\right. \\
& \left.+\frac{a^{3}\left(3 a^{2}-2 b^{2}\right)^{2}}{(2 b+3 a)^{6}}-\ldots\right] \frac{\mathrm{P}_{3}(\cos \theta)}{r^{4}} \\
& +4 b^{3}\left[a^{3}-\frac{a^{5}\left(b^{2}+a b-a^{2}\right)^{3}}{(b+a)^{6}}+\frac{a^{3}\left(2 a^{2}-b^{2}\right)^{3}}{(b+2 a)^{6}}-\frac{a^{3}\left(2 b^{2}+a b-2 a^{2}\right)^{3}}{(2 b+2 a)^{6}}\right. \\
& \left.+\frac{a^{3}\left(3 a^{2}-2 b^{2}\right)^{3}}{(2 b+3 a)^{6}}-\ldots\right] \frac{\mathrm{P}_{4}(\cos \theta)}{r^{5}} \\
& \text { - \&c., . . . . . . }
\end{aligned}
$$

the law of formation of the series within the brackets being obvious.

Coming now to the present problem of two unequal spheres of the same material, let us take

$$
a=2 \text { inches and } b=1 \text { inch. }
$$

Since the changes of velocities of the two spheres are inversely proportional to their masses, we must have

$$
\mathrm{U}_{b}=8 \mathrm{U}_{a} \text {. }
$$


Substituting the values for $a$ and $b$, we easily find that

$$
\begin{aligned}
& 2 \phi=2^{3}\left[\left(1+\frac{1}{4^{3}}+\frac{1}{7^{3}}+\frac{1}{10^{3}}+\ldots\right)\right. \\
& \left.-\left(\frac{1}{3^{3}}+\frac{1}{6^{3}}+\frac{1}{9^{3}}+\ldots\right)\right] \frac{P_{1}(\cos \theta)}{r^{2}} \\
& +2.2^{3}\left[1-\left(\frac{5}{3^{4}}+\frac{8}{6^{4}}+\frac{11}{9^{4}}+\frac{14}{12^{4}}+\ldots\right)\right. \\
& \left.-\left(\frac{2}{4^{4}}+\frac{5}{7^{4}}+\frac{8}{10^{4}}+\ldots\right)\right] \frac{\mathrm{P}_{2}(\cos \theta)}{r^{3}} \\
& +3.2^{3}\left[\left(1+\frac{2^{2}}{4^{5}}+\frac{5^{2}}{7^{5}}+\frac{8^{2}}{10^{5}}+\ldots\right)\right. \\
& \left.-\left(\frac{5^{2}}{3^{5}}+\frac{8^{2}}{6^{5}}+\frac{11^{2}}{9^{5}}+\ldots\right)\right] \frac{P_{3}(\cos \theta)}{r^{4}} \\
& +4.2^{3}\left[1-\left(\frac{5^{3}}{3^{6}}+\frac{8^{3}}{6^{6}}+\frac{11^{3}}{9^{6}}+\frac{14^{3}}{12^{6}}+\ldots\right)\right. \\
& \left.-\left(\frac{2^{3}}{4^{6}}+\frac{5^{3}}{7^{0}}+\frac{8^{3}}{10^{6}}+\ldots\right)\right] \frac{\mathrm{P}_{4}(\cos \theta)}{r^{3}} \\
& \text { +\&c., . . . . . . }
\end{aligned}
$$

and

$$
\begin{aligned}
& 2 \phi^{\prime}=-2^{3}\left[\left(\frac{1}{2^{3}}+\frac{1}{5^{3}}\right.\right.\left.+\frac{1}{8^{3}}+\frac{1}{11^{3}}+\ldots\right) \\
&\left.-\left(\frac{1}{3^{3}}+\frac{1}{6^{3}}+\frac{1}{9^{3}}+\ldots\right)\right] \frac{\mathrm{P}_{1}(\cos \theta)}{r^{2}} \\
&+2.2^{3}\left[\left(\frac{1}{3^{4}}+\frac{4}{6^{4}}+\frac{7}{9^{4}}+\ldots\right)\right. \\
&\left.+\left(\frac{4}{2^{4}}+\frac{7}{5^{4}}+\frac{10}{8^{4}}+\frac{13}{11^{4}}+\ldots\right)\right] \frac{P_{2}(\cos \theta)}{r^{3}} \\
&-3.2^{3}\left[\left(\frac{4^{2}}{2^{5}}+\frac{7^{2}}{5^{5}}+\frac{10^{2}}{8^{5}}+\frac{13^{2}}{11^{5}}+\ldots\right)\right. \\
&\left.-\left(\frac{1^{2}}{3^{5}}+\frac{4^{2}}{6^{5}}+\frac{7^{2}}{9^{5}}+\ldots\right)\right] \frac{\mathrm{P}_{3}(\cos \theta)}{r^{4}}
\end{aligned}
$$$$
+4.2^{3}\left[\left(\frac{1^{3}}{3^{6}}+\frac{4^{3}}{6^{6}}+\frac{7^{3}}{9^{6}}+\ldots\right)\right.
$$$$
\left.+\left(\frac{4^{3}}{2^{6}}+\frac{7^{3}}{5^{6}}+\frac{10^{3}}{8^{5}}+\frac{13^{3}}{11^{8}}+\ldots\right)\right] \frac{\mathrm{P}_{4}(\cos \theta)}{r^{5}}
$$$$
\text { - \&c. . . . . . . }
$$ 
Summing the series, we easily find that the vibration on the surface of the enveloping sphere

$$
\begin{aligned}
&= \mathrm{U}_{a} \\
& {\left[\frac{\partial \phi}{\partial r}+8 \frac{\partial \phi^{\prime}}{\partial r}\right] e_{r=3 \text { inches }}^{i k c t} } \\
&=-\frac{4}{27} \mathrm{U}_{a}\left[\cdot 496 \mathrm{P}_{1}(\cos \theta)+3 \cdot 180 \mathrm{P}_{2}(\cos \theta)\right. \\
& \\
&\left.\quad-1 \cdot 708 \mathrm{P}_{3}(\cos \theta)+2 \cdot 600 \mathrm{P}_{4}(\cos \theta)+\ldots\right] e^{i k c t}
\end{aligned}
$$

We have seen that when the vibration on the surface of the enveloping sphere is

$$
\sum \mathbf{A}_{n} P_{n}(\cos \theta) \cdot \epsilon^{i k c t}
$$

the velocity potential of the wave-disturbance is

$$
\psi=-\frac{(a+b)^{2}}{r} e^{i k(c t-r+a+b)} \sum \frac{A_{n} \mathrm{P}_{n}(\cos \theta)}{\mathrm{F}_{n}(i k . \overline{a+b})} J_{n}(i k r) .
$$

Now when $r$ is large, $f_{n}(i k r)=1$, so that the factor on which the relative intensities in various directions depend is

$$
\Sigma \mathbf{A}_{n} \frac{\mathbf{P}_{n}(\cos \theta)}{\mathbf{F}_{n}(i k \cdot \overline{a+b})} \text {. }
$$

Thus if we put this quantity $=F+i G$, the intensity of the vibrations in various directions is measured by $\mathrm{F}^{2}+\mathrm{G}^{2}$.

The distribution of intensities in different directions round the spberes will be influenced to a considerable extent by the value of the wave-length chosen. If we take $k(a+b)=2$, the wave-length is $3 \pi$ inches, and if we take $k(a+b)=3$, the wave-length is $2 \pi$ inches. From the expression (1) for the wave-motion produced by a single sphere undergoing an instantaneous change of velocity, it is seen that the wavelength to be chosen is of the same order as the circumference of the sphere. From this, it appears that for a system of two spheres whose radii are 1 inch and $z$ inches respectively, the wave-length to be chosen should be some value intermediate between $2 \pi$ and $4 \pi$, probably nearer $2 \pi$ than $4 \pi$; for, in the actual case of impact, the smaller ball which would undergo by far the greater change in velocity would probably influence the character of the motion to a greater extent than the larger sphere. At the same time, it must not be forgotten that the analogy between the cases of impact and of periodic motion cannot be pushed very far, inasmuch as the fluid motion due to impact is undoubtedly 
of different character in different directions, and not all throughout the same as in the periodic case.

Now taking $k(a+b)=2$, we find (neglecting a constant factor)

$$
\begin{array}{r}
\mathrm{F}=.0992 \mathrm{P}_{1}(\cos \theta)+\cdot 2840 \mathrm{P}_{2}(\cos \theta)-\cdot 0354 \mathrm{P}_{3}(\cos \theta) \\
-\cdot 0146 \mathrm{P}_{4}(\cos \theta)+\& \mathrm{c} ., \\
\begin{aligned}
\mathrm{G}=.0496 \mathrm{P}_{1}(\cos \theta) & -\cdot 4040 \mathrm{P}_{2}(\cos \theta)-\cdot 0177 \mathrm{P}_{3}(\cos \theta) \\
& +.0315 \mathrm{P}_{4}(\cos \theta)+\& c . \quad . \quad . \quad .
\end{aligned}
\end{array}
$$

The values of $F$ and $G$ for different directions have been calculated and are shown in the following table:-

\section{Table I.}

\begin{tabular}{cccc}
$\begin{array}{c}\text { Angles } \\
\text { (in degrees). }\end{array}$ & F $\times$ oonst. & $G \times$ eonst. & $\left(\mathrm{F}^{2}+\mathrm{G}^{2}\right) \times$ const. \\
\hline 0 & +329000 & -338000 & 223144 \\
10 & +325908 & -326525 & 212552 \\
20 & +297435 & -273545 & 162738 \\
30 & +251537 & -214337 & 109300 \\
40 & +189095 & -114659 & 48946 \\
50 & +114215 & -24167 & 13572 \\
60 & +33341 & +74407 & 6565 \\
70 & -43647 & +155306 & 25961 \\
80 & -107073 & +205212 & 53574 \\
90 & -147250 & +213625 & 67405 \\
100 & -158815 & +178920 & 57322 \\
110 & -140329 & +106238 & 30836 \\
120 & -95149 & +8675 & 9106 \\
130 & -34099 & -99267 & 10957 \\
140 & +35677 & -202159 & 42100 \\
150 & +102819 & -289237 & 94130 \\
160 & +157869 & -353605 & 150280 \\
170 & +192648 & -392229 & 190913 \\
180 & +201000 & -404000 & 203617
\end{tabular}

Now taking $k(a+b)=3$, we find (neglecting a constant factor)

$$
\begin{array}{r}
\mathrm{F}=\begin{array}{r}
105 \mathrm{P}_{1}(\cos \theta)+1 \cdot 060 \mathrm{P}_{2}(\cos \theta)+\cdot 016 \mathrm{P}_{3}(\cos \theta) \\
-\cdot 281 \mathrm{P}_{4}(\cos \theta)-\& c .
\end{array} \\
\mathrm{G}=-\cdot 122 \mathrm{P}_{1}(\cos \theta)+\cdot 186 \mathrm{P}_{3}(\cos \theta)-.024 \mathrm{P}_{4}(\cos \theta) \\
- \text { \&c. . . . . . . . . . . }
\end{array}
$$


The values in different directions have been calculated from these expressions and are shown in the following table :-

\section{TABLE II.}

\begin{tabular}{|c|}
\hline Angles \\
\hline
\end{tabular}

$\begin{array}{rrrr}0 & +900000 & +38000 & 811444 \\ 10 & +890608 & +28804 & 794265 \\ 20 & +849305 & -2390 & 720805 \\ 30 & +752167 & -45754 & 567620 \\ 40 & +572469 & -90446 & 335284 \\ 50 & +309902 & -123998 & 111476 \\ 60 & -5783 & -135346 & 18261 \\ 70 & -313014 & -118446 & 111893 \\ 80 & -542728 & -73554 & 300178 \\ 90 & -635375 & -9000 & 403306 \\ 100 & -571364 & +60786 & 329762 \\ 110 & -371618 & +118638 & 154545 \\ 120 & -96799 & +149218 & 31610 \\ 130 & +184472 & +144494 & 54592 \\ 140 & +412409 & +105758 & 180980 \\ 150 & +559907 & +44650 & 315625 \\ 160 & +630625 & -21410 & 398602 \\ 170 & +654606 & -69748 & 433925 \\ 180 & +658000 & -88000 & 440708\end{array}$

The values of $\mathrm{F}^{2}+\mathrm{G}^{2}$ shown in Tables $\mathrm{I}$. and II., have been plotted in polar coordinates in figs. 3 and 4 (PI. IV.). It is seen that in both cases the intensity in the direction of the larger ball is greater than in the direction of the smaller ball. The asymmetry is more marked when $k(a+b)$ has the larger value.

The intensity of the sound in different directions due to the impact of two spheres of wood of diameters 3 inches and $1 \frac{1}{2}$ inches respectively has been measured with the ballistic phonometer and is shown in fig. 5. It is seen that this curve is intermediate in form between those shown in fig. 3 and fig. 4, exactly as anticipated. The agreement between theory and experiment is thus very striking in this case. 


\section{Two spheres of the same diameter but of different materials.}

We have seen in the preceding section that in the expressions for $F$ and $G$ for two spheres of the same material but of unequal diameters, the terms containing the zonal harmonic of the second order $\mathrm{P}_{2}(\cos \theta)$ usually preponderate, and that the intensity diagram is, accordingly, a curve which consists of four loops. A different result is obtained in the case of two spheres of the same diameter but of markedly unequal densities. The zonal harmonic of the first order preponderates in this case, and the intensity diagram is a curve consisting of only two loops. To obtain this result theoretically, we have to proceed on exactly the same lines as in the preceding pages.

Taking $a=1$ inch and $b=1$ inch, we easily find from the expressions (7) and (8) that

$$
\begin{array}{r}
2 \phi=\left[1-\frac{1}{2^{3}}+\frac{1}{3^{3}}-\frac{1}{4^{3}}+\frac{1}{5^{3}}-\ldots\right] \frac{\mathrm{P}_{1}(\cos \theta)}{r^{2}} \\
+2\left[1-\frac{1}{2^{4}}+\frac{1}{3^{4}}-\frac{1}{4^{4}}+\frac{1}{5^{4}}-\ldots\right] \frac{\mathrm{P}_{2}(\cos \theta)}{r^{3}} \\
+3\left[1-\frac{1}{2^{5}}+\frac{1}{3^{5}}-\frac{1}{4^{5}}+\frac{1}{5^{5}}-\ldots\right] \frac{\mathrm{P}_{3}(\cos \theta)}{r^{4}} \\
+4\left[1-\frac{1}{2^{8}}+\frac{1}{3^{6}}-\frac{1}{4^{6}}+\frac{1}{5^{6}}-\ldots\right] \frac{\mathrm{P}_{4}(\cos \theta)}{r^{5}} \\
+ \text { \&c., . . . . . . }
\end{array}
$$

and

$$
\begin{aligned}
2 \phi^{\prime}= & -\left[1-\frac{1}{2^{3}}+\frac{1}{3^{3}}-\frac{1}{4^{3}}+\frac{1}{5^{3}}-\ldots\right] \frac{\mathrm{P}_{1}(\cos \theta)}{r^{2}} \\
& +\left[1-\frac{1}{2^{4}}+\frac{1}{3^{4}}-\frac{1}{4^{4}}+\frac{1}{5^{4}}-\ldots\right] \frac{\mathrm{P}_{2}(\cos \theta)}{r^{3}} \\
& -\left[1-\frac{1}{2^{5}}+\frac{1}{3^{5}}-\frac{1}{4^{5}}+\frac{1}{5^{5}}-\ldots\right] \frac{\mathrm{P}_{3}(\cos \theta)}{r^{4}} \\
& +\left[1-\frac{1}{2^{8}}+\frac{1}{3^{6}}-\frac{1}{4^{6}}+\frac{1}{5^{6}}-\ldots\right] \frac{\mathrm{P}_{4}(\cos \theta)}{r^{5}} \\
& -\& c . . . . . .
\end{aligned}
$$


Summing the series, we find that the vibrations on the surface of the enveloping sphere, namely

$$
\left[\mathrm{U}_{a} \frac{\partial \phi}{\partial r}+\mathrm{U}_{b} \frac{\partial \phi^{\prime}}{\partial r}\right] e_{r=2 \text { inches }}^{i k e t}
$$

can be expressed in the form

$$
\begin{aligned}
& \frac{1}{2}\left[\left(\mathrm{U}_{a}-\mathrm{U}_{b}\right) \times \cdot 2254 \mathrm{P}_{1}(\cos \theta)+\left(\mathrm{U}_{a}+\mathrm{U}_{b}\right) \times \cdot 3550 \mathrm{P}_{2}(\cos \theta)\right. \\
& +\left(\mathrm{U}_{a}-\mathrm{U}_{b}\right) \times \cdot 3645 \mathrm{P}_{3}(\cos \theta)+\left(\mathrm{U}_{a}+\mathrm{U}_{b}\right) \times \cdot 3080 \mathrm{P}_{4}(\cos \theta) \\
& \left.+\left(\mathrm{U}_{a}-\mathrm{U}_{b}\right) \times \cdot 2325 \mathrm{P}_{5}(\cos \theta)+\& \mathrm{c} .\right] e^{i k t} \cdot . \quad \cdot . \quad \cdot(16)
\end{aligned}
$$

If the ball of radius $b$ is four times heavier than the one of radius $a$, we have

$$
\mathrm{U}_{a}=4 \mathrm{U}_{b} .
$$

So that the vibration on the surface of the enveloping sphere is proportional to the expression

$$
\begin{aligned}
.6762 \mathrm{P}_{1}(\cos \theta) & +1.7750 \mathrm{P}_{2}(\cos \theta)+1.0935 \mathrm{P}_{3}(\cos \theta) \\
& +1.5400 \mathrm{P}_{4}(\cos \theta)+.6975 \mathrm{P}_{5}(\cos \theta)+\& \mathrm{c} .
\end{aligned}
$$

Now taking $k(a+b)=1$, which will give a wave-length equal to the circumference of the enveloping sphere, we get (neglecting a constant factor)

$$
\begin{array}{r}
\mathrm{F}=\quad .13524 \mathrm{P}_{1}(\cos \theta)-.04987 \mathrm{P}_{2}(\cos \theta)-\cdot 0074 \mathrm{P}_{3}(\cos \theta) \\
+\cdot 0007 \mathrm{P}_{4}(\cos \theta)+\& c . \\
\mathrm{G}=-.0676 \mathrm{P}_{1}(\cos \theta)-.0798 \mathrm{P}_{2}(\cos \theta)+\cdot 0047 \mathrm{P}_{3}(\cos \theta) \\
+\cdot 0012 \mathrm{P}_{4}(\cos \theta)-\& c . \quad \cdot \quad \cdot(17)
\end{array}
$$

The values of $\mathrm{F}$ and $\mathrm{G}$, and of $\mathrm{F}^{2}+\mathrm{G}^{2}$ in different directions obtained from the preceding expressions are shown in Table III.

The values of $\left(\mathrm{F}^{2}+\mathrm{G}^{2}\right)$ shown in Table III. have been plotted in polar coordinates and are shown in fig. 6 . It is seen that the maximum intensity in the direction of the heavier ball is greater than that in the direction of the lighter one.

The experimental curve of intensity of sound due to impact of a sphere of wood, diameter $2 \frac{1}{4}$ inches, with a billiard ball of nearly the same size is shown in fig. 7 . It is found 
that the directions of minimum intensity are not quite in the plane perpendicular to the line of impact, being nearer the side of the lighter ball.

TABLe III.

$\underset{\text { (in degrees). }}{\text { Angles }} \quad F \times$ const. $\quad G \times$ const. $\quad\left(F^{2}+G^{2}\right) \times$ const.

$\begin{array}{rrrr}0 & +786000 & -1415000 & 2620021 \\ 10 & +793732 & -1374897 & 2521061 \\ 20 & +813819 & -1256037 & 2240132 \\ 30 & +835068 & -1068615 & 1839986 \\ 40 & +845629 & -826059 & 1397992 \\ 50 & +828667 & -549652 & 989741 \\ 60 & +768690 & -262257 & 660005 \\ 70 & +654594 & +7901 & 427780 \\ 80 & +482433 & +237049 & 288493 \\ 90 & +252125 & +403500 & 225913 \\ 100 & +228907 & +495515 & 297466 \\ 110 & -331298 & +509107 & 368642 \\ 120 & -647986 & +454821 & 626929 \\ 130 & -954405 & +347884 & 1031220 \\ 140 & -1229335 & +211923 & 1555385 \\ 150 & -1458496 & +71667 & 2130948 \\ 160 & -1629521 & -47667 & 2655850 \\ 170 & -1734880 & -128811 & 3026866 \\ 180 & -1770000 & -157000 & 3157549\end{array}$

A result of some importance indicated by theory is that when one of the spheres is much heavier than the other, replacing the former by a still heavier sphere of the same diameter should not result in any important alteration in the distribution of the intensity of sound in different directions due to impact. This is clear from expression (16). For when $\mathrm{U}_{a}$ is much larger than $\mathrm{U}_{b}$, any diminution in the value of $\mathrm{U}_{b}$ should not appreciably affect the value of the expression. This indication of theory is in agreement with experiment. Several series of measurements have been made with various pairs of balls of the same size but of different densities, e.g., wood and marble, wood and iron, billiard ball and iron ball, and so forth. Generally, similar results are obtained in all cases. It was noticed also that the form of the intensity distribution as shown by the ballistic phonometer was not altogether independent of the thickness of the mica disk used in the instrument. This is not surprising, as the behavionr of the mica disk before the pointer 
attached to it ceases to touch the mirror of the indicator would no doubt depend, to some extent, on the relation between its natural frequency and the frequency of the sound-waves set up by the impact. The best results were obtained with a disk neither so thick as to be relatively insensitive nor so thin as to remain with its pointer in contact with the indicator longer than absolutely necessary.

\section{The general case of spheres of any diameter and density.}

When the impinging spheres are both of different diameter and of different density, the result generally obtained is that the sound is a maximum on the line of impact in either direction, and a minimum which approaches zero in directions asymmetrically situated with reference thereto. Generally speaking, no maxima in lateral directions are noticed, that is, the curve consists of two nearly closed loops. The difference of the intensity of the sound in the two directions of the line of impact may sometimes be very considerable. As a typical case, the results obtained by the impact of a sphere of wood 3 inches in diameter with a brass sphere only $1 \frac{1}{8}$ inch in diameter are shown in fig. 8 . It is observed that the sound due to impact is actually of greater intensity on the side of the small brass ball. As a matter of fact, the result generally obtained is that the intensity is greater on the side of the ball of the denses material even if its diameter be the smaller.

The mathematical treatment of the general case is precisely on the same lines as in the two preceding sections. It is found in agreement with the experimental result that in practically all cases in which both the densities and the diameters are different, the zonal harmonic of the first order is of importance and that the intensity curve consists of two nearly closed loops, as in the case of two spheres of the same diameter but of different density.

\section{Summary and Conclusion.}

The investigation of the origin and characteristics of the sound due to the direct impact of two similar solid spheres which was described in the Phil. Mag. for July, 1916, has been extended in the present paper to the cases in which the impinging spheres are not both of the same diameter or 
material. The relative intensities of the sound in different directions have been measured by the aid of the ballistic phonometer, and in order to exhibit the results in an effective manner, they have been plotted in polar coordinates, the point at which the spheres impinge being taken as the origin, and the line of collision as the axis of $x$. As might be expected, the curves thus drawn show marked asymmetry in respect of the plane perpendicular to the line of impact.

A detailed mathematical discussion of the nature of the results to be expected is possible by considering the analogons case of two rigid spheres nearly in contact which vibrate bodily along their line of centres. By choosing an appropriate wave-length for the resulting motion, intensity curves similar to those found experimentally for the case of impact are arrived at. A further confirmation is thus obtained of the hypothesis regarding the origin of the sound suggested by the work of Hertz and of Lord Rayleigh on the theory of elastic impact.

When the impinging spheres, though not equal in size, are of the same or nearly the same density, the intensity-curve drawn for the plane of observation shows the sound to be a maximum along the line of impact in either direction, and also along two directions making equal acute angles with this line. The sound is a minimum along four directions in the plane. In practically all other cases, that is when the spheres differ considerably either in density alone, or both in diameter and density, the intensity is found to be a maximum along the line of impact in either direction, and to be a minimuin along directions which are nearly but not quite perpendicular to the line of impact. The form of the intensity curve is practically determined by the diameters and the masses of the spheres.

The investigation was carried out in the Physical Laboratory of the Indian Association for the Cultivation of Science. It is hoped when a suitable opportunity arises to study also the case of oblique impact. 'The writer has much pleasure in acknowledging the helpful interest taken by Prof. C. V. Raman in the progress of the work described in the present paper.

Calcutta,

15th June, 1917. 


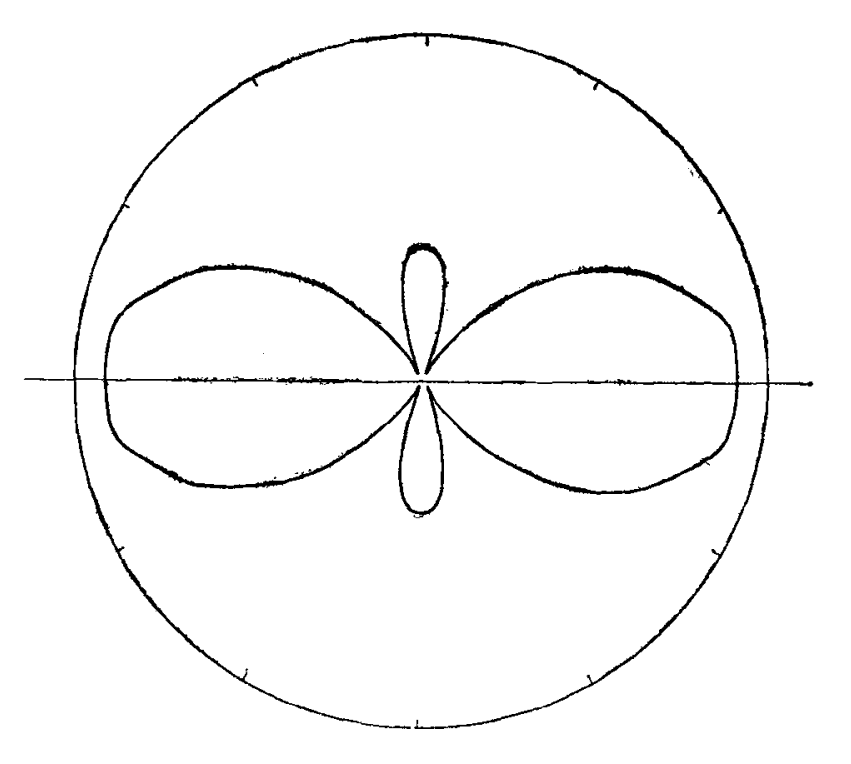

FiG. 1-Observed distribution of sound intonsity around two
equal colliding spheres.

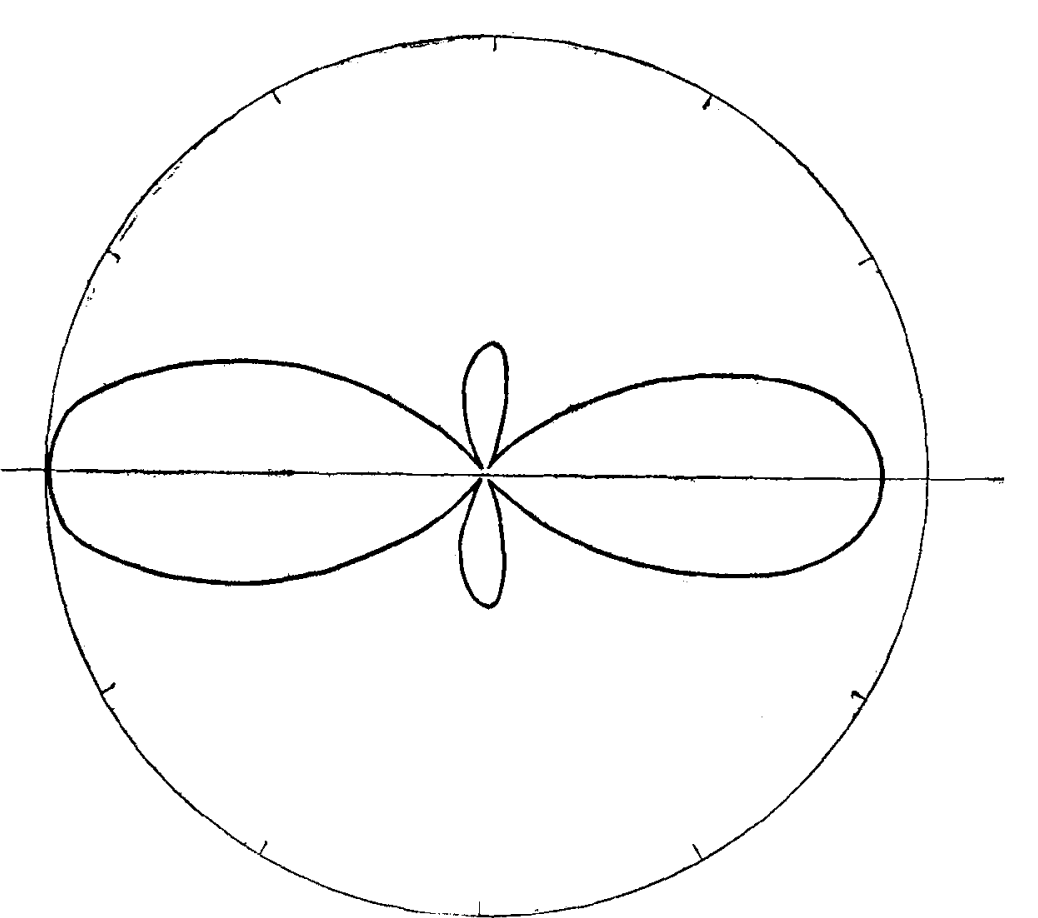

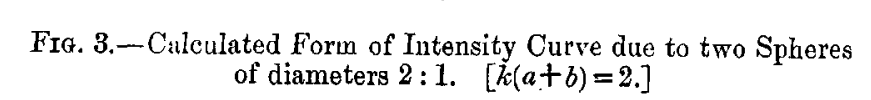

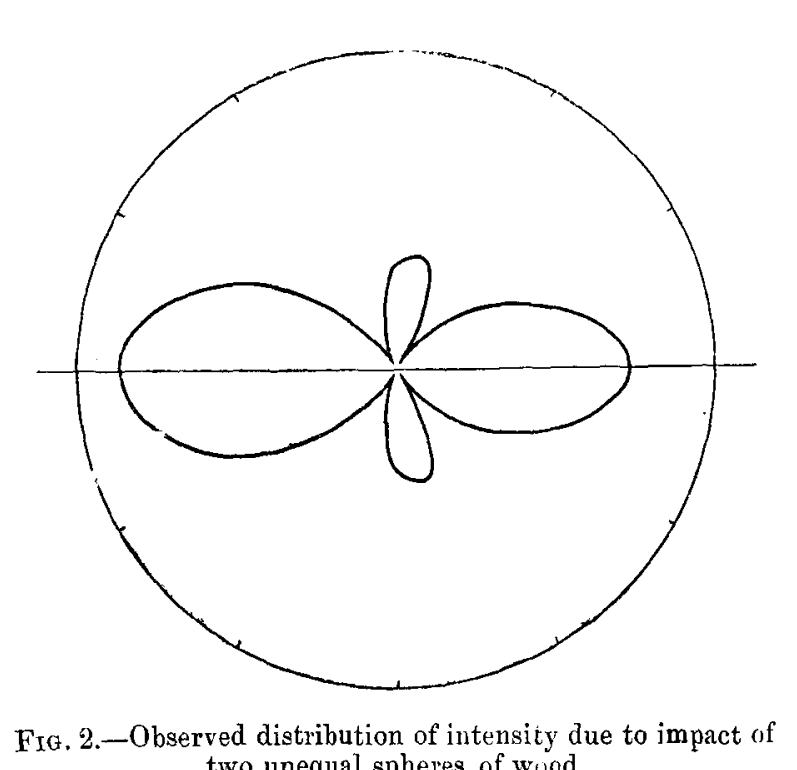

Sphere on lett;
3 inches diameter. Sphere on right;
2f inclies diameter.

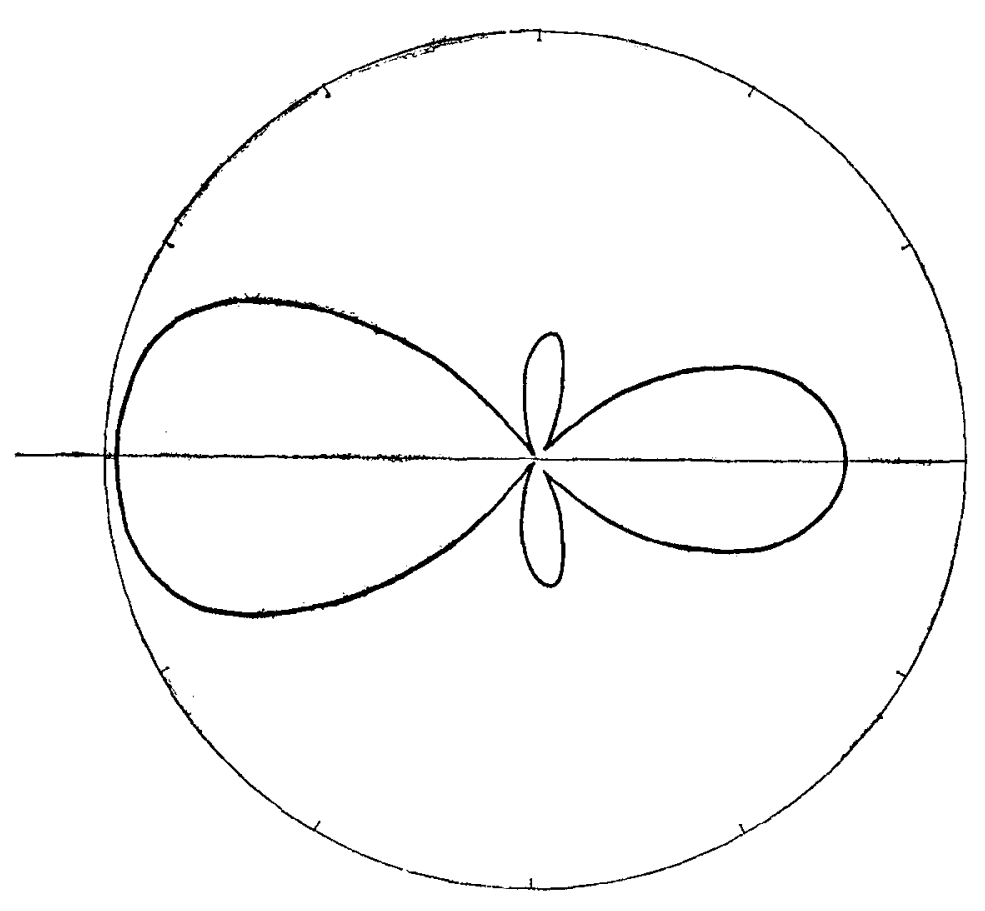

FIG. 5.-Observed Form of Intensity Curre due to impact of (Material, wood; diameeters 33 inches and 1 is inches respectively.)

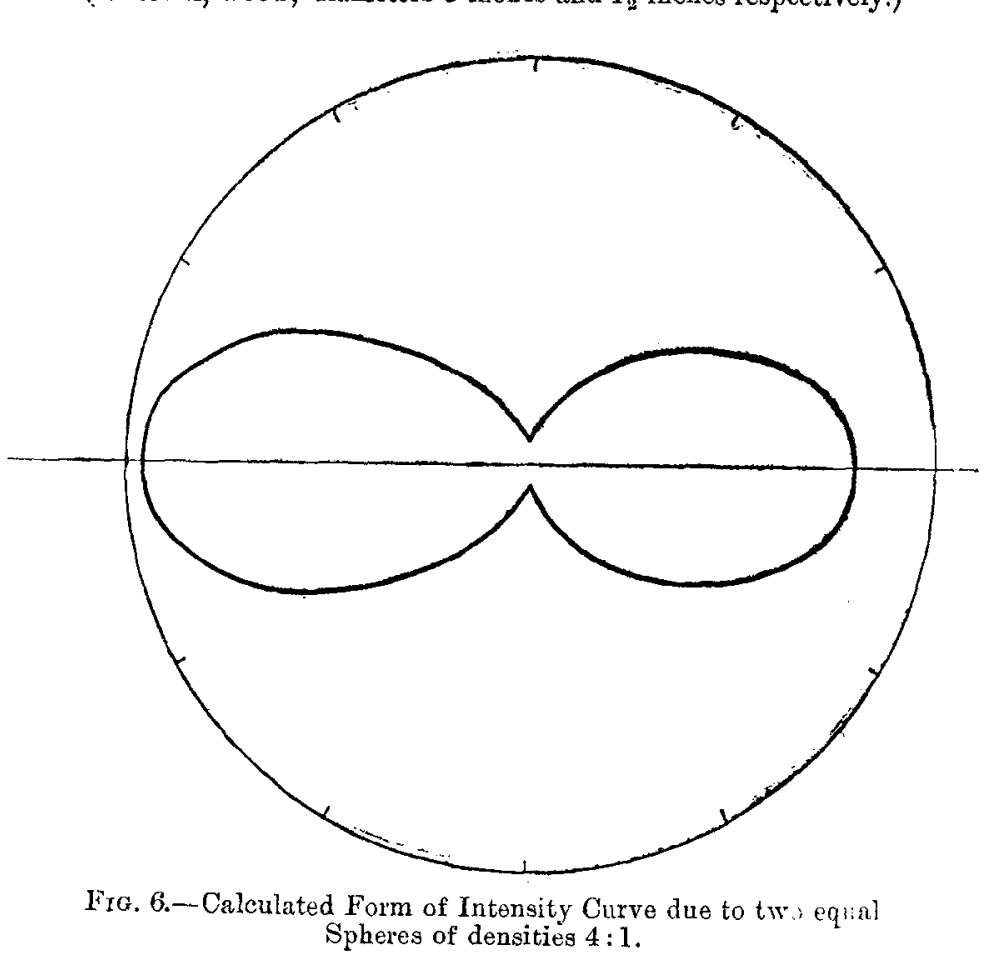

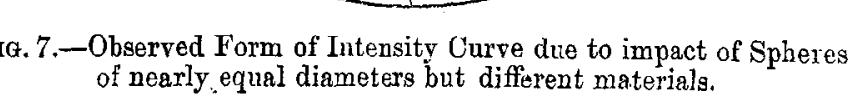

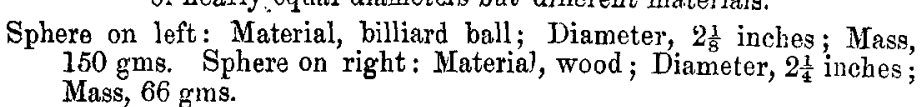

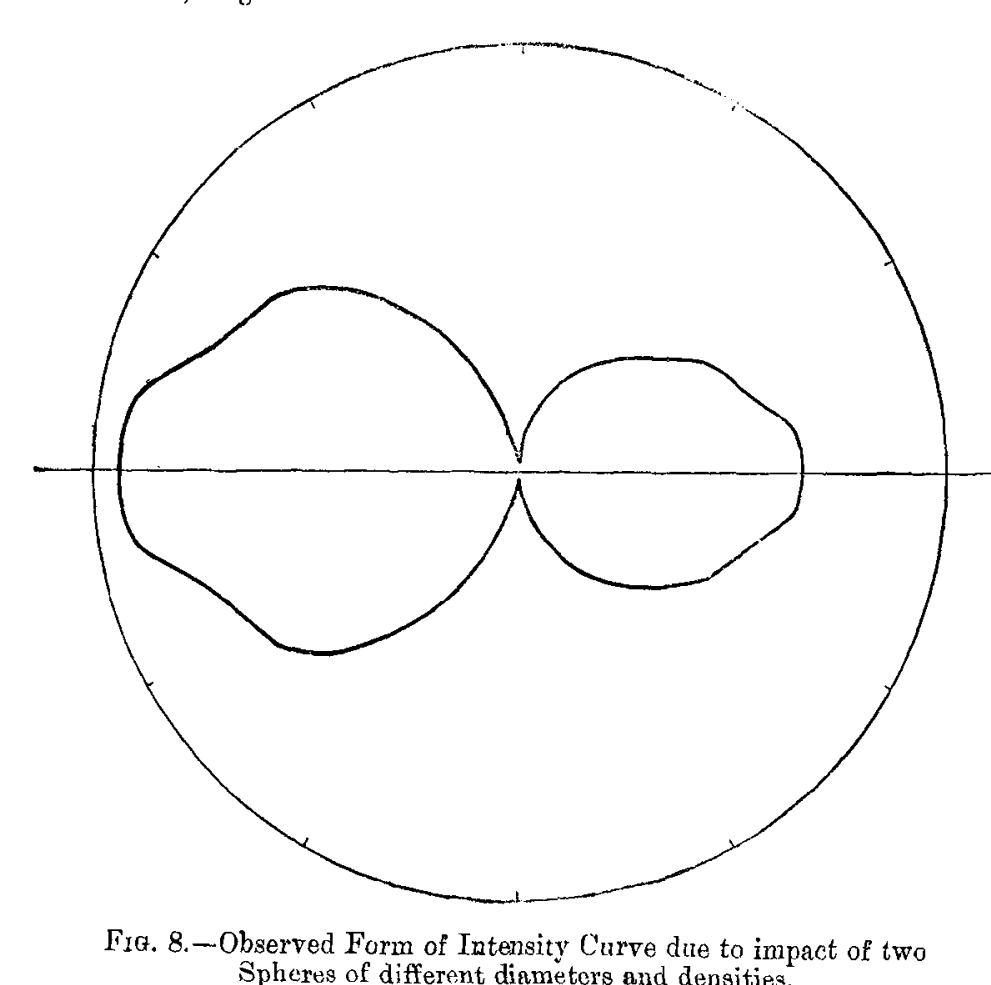

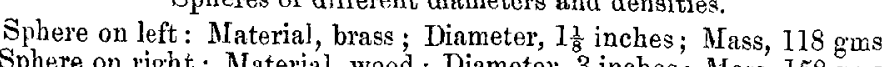

